\title{
Functional Organization of Visual Cortex in the Owl Monkey
}

\author{
Xiangmin Xu, ${ }^{1}$ William Bosking, ${ }^{2}$ Gyula Sáry, ${ }^{3,4}$ James Stefansic, ${ }^{1}$ Daniel Shima, ${ }^{1}$ and Vivien Casagrande ${ }^{1,3}$ \\ ${ }^{1}$ Department of Psychology, Vanderbilt University, Nashville, Tennessee 37232-2175, ${ }^{2}$ Division of Neuroscience and Howard Hughes Medical Institute, \\ Baylor College of Medicine, Houston, Texas 77030, ${ }^{3}$ Department of Cell and Developmental Biology, Vanderbilt University, Nashville, Tennessee $37232-$ \\ 2175, and ${ }^{4}$ Department of Physiology, Faculty of Medicine, University of Szeged, Szeged, Hungary, H-6720
}

In this study, we compared the organization of orientation preference in visual areas $\mathrm{V} 1, \mathrm{~V} 2$, and $\mathrm{V} 3$. Within these visual areas, we also quantified the relationship between orientation preference and cytochrome oxidase (CO) staining patterns. V1 maps of orientation preference contained both pinwheels and linear zones. The location of $\mathrm{CO}$ blobs did not relate in a systematic way to maps of orientation; although, as in other primates, there were approximately twice as many pinwheels as CO blobs. V2 contained bands of high and low orientation selectivity. The bands of high orientation selectivity were organized into pinwheels and linear zones, but iso-orientation domains were twice as large as those in V1. Quantitative comparisons between bands containing high or low orientation selectivity and CO dark and light bands suggested that at least four functional compartments exist in V2, CO dense bands with either high or low orientation selectivity, and $\mathrm{CO}$ light bands with either high or low selectivity. We also demonstrated that two functional compartments exist in $\mathrm{V}$, with zones of high orientation selectivity corresponding to $\mathrm{CO}$ dense areas and zones of low orientation selectivity corresponding to $\mathrm{CO}$ pale areas. Together with previous findings, these results suggest that the modular organization of $\mathrm{V} 1$ is similar across primates and indeed across most mammals. V2 organization in owl monkeys also appears similar to that of other simians but different from that of prosimians and other mammals. Finally, V3 of owl monkeys shows a compartmental organization for orientation selectivity that remains to be demonstrated in other primates.

Key words: optical imaging; owl monkey; striate cortex; extrastriate; cytochrome oxidase; orientation selectivity

\section{Introduction}

A common feature of early visual areas is the existence of modules wherein cells that have similar functional properties are grouped together. For example, single-cell recordings, metabolic labeling, and optical imaging have demonstrated that visual cortical neurons with similar orientation selectivity tend to group together in columns (Hubel and Wiesel, 1977; Blasdel and Salama, 1986; Roe and Ts'o, 1995; Vanduffel et al., 2002). Additionally, functional modules often have been defined in relationship to compartments stained by cytochrome oxidase (CO) (e.g., CO blobs within V1, and CO stripes in V2) (Horton and Hubel, 1981; Livingstone and Hubel, 1984; Sincich and Horton, 2002). The relationship of orientation selective cells relative to CO compartments, however, has been the subject of considerable controversy. This is especially true for V1, with some investigators supporting a strong relationship, and others finding no relationship (Livingstone and Hubel, 1984; DeYoe and Van Essen, 1985; Ts'o and Gilbert, 1988; Lennie et al., 1990; DeBruyn et al., 1993; Edwards et al., 1995; Leventhal et al., 1995; Roe and Ts'o, 1995;

Received Nov. 22, 2003; revised May 20, 2004; accepted May 24, 2004.

This work was supported by National Institutes of Health (NIH) Grant EY01778 (V.A.C.), NIH Core Grants EY08126 and HD 15052, and National Center for Research Resources Shared Instrumentation Grant 1S10RR13947 (V.A.C.). We thank Anna Roe, Jon Kaas, Ford Ebner, Jeffrey Schall, René Marois, and Julie Mavity-Hudson for helpful comments on this manuscript. We also thank Julie Mavity-Hudson for excellent technical assistance and John Allison and Mary Feurtado for help with animal preparation.

Correspondence should be addressed to Dr. V.A. Casagrande, Department of Cell and Developmental Biology, Vanderbilt Medical School, Medical Center North B2323, Nashville, TN 37232-2175. E-mail: vivien.casagrande@vanderbilt.edu. DOl:10.1523/JNEUROSCI.1144-04.2004

Copyright $\odot 2004$ Society for Neuroscience $\quad 0270-6474 / 04 / 246237-11 \$ 15.00 / 0$
Landisman and Ts'o, 2002a,b). Furthermore, quantification of the differences in orientation selectivity between compartments have usually been limited to assignment of single cells to one of a number of arbitrary groups or by subjective evaluation of the relationship between maps derived by optical imaging and staining for CO (Livingstone and Hubel, 1984; Ts'o et al., 1990; Malach et al., 1994). Although there have been many studies that have investigated the functional organization of $\mathrm{V} 2$, most of these were limited to macaque monkeys and were quantified in a similar manner (for review, see Roe and Ts'o, 1997). Finally, only a few investigations have been made of the functional architecture of V3, and no studies have convincingly demonstrated separate functional compartments within this area in any species (Felleman and Van Essen, 1987; Gegenfurtner et al., 1997; Adams and Zeki, 2001).

In this study, we examined and compared the functional organization of orientation preference of V1, V2, and V3 using optical imaging of intrinsic signals and quantitatively examined relationships between orientation preference domains and $\mathrm{CO}$ modules in these cortical areas. Owl monkeys offered several advantages for these studies. First, with the exception of macaque monkeys, the visual system of owl monkeys has been studied in the most detail (for review, see Casagrande and Kaas, 1994). Second, owl monkeys have relatively small lissencephalic brains. This feature allowed us to easily access extrastriate areas for optical imaging. Third, owl monkeys are of special interest both because they are the only nocturnal simians in existence, and because they have only a single cone type and thus lack color vision. Finally, despite these differences from other simians, they 
have well defined CO modules in many visual areas (e.g., V1, V2, and V3) (Tootell et al., 1985; Wikler and Rakic, 1990; Casagrande and Kaas, 1994; Jacobs et al., 1996; Lyon and Kaas, 2002).

\section{Materials and Methods}

General preparation. The 10 owl monkeys used in this study were handled according to an approved protocol from the Vanderbilt University Animal Care and Use Committee. Animals were prepared for surgery, paralyzed, and anesthetized as described in detail previously (Xu et al., 2001; $\mathrm{Xu}, 2004)$. In these animals, V1 was imaged in 10 hemispheres, V2 in 10 hemispheres, and V3 in five hemispheres.

The animals were initially anesthetized with isoflurane $\left(2-4 \%\right.$ in $\left.\mathrm{O}_{2}\right)$ and, after tracheal intubation and implantation with a femoral catheter, were mounted in a stereotaxic apparatus. Neuromuscular blockade was initiated by intravenous injection of $1-1.5 \mathrm{mg} \mathrm{kg}^{-1}$ vecuronium bromide. Animals were then artificially ventilated with a mixture of $75 \%$ $\mathrm{N}_{2} \mathrm{O}, 23.5 \% \mathrm{O}_{2}$, and $1.5 \% \mathrm{CO}_{2}$ delivered at a rate sufficient to maintain the peak end tidal $\mathrm{CO}_{2}$ level at $\sim 4 \%$. In most cases, paralysis and anesthesia were maintained by intravenous infusion of vecuronium bromide (0.1-0.2 $\mathrm{mg} \mathrm{kg} / \mathrm{hr}$ ) and sufentanil citrate (Sufenta, $12-15 \mathrm{mg} \mathrm{kg} / \mathrm{hr}$ ) mixed in $5 \%$ dextrose lactated Ringer's delivered at a rate of $\sim 2.7 \mathrm{ml} / \mathrm{hr}$. For a minority of cases, anesthesia was maintained by intravenous infusion of propofol (2,6-di-isopropylphenol, $10 \mathrm{mg} \mathrm{kg} / \mathrm{hr}$ ) through a separate infusion line. To ensure that adequate levels of anesthesia were maintained throughout the experiment, heart rate, peak end tidal $\mathrm{CO}_{2}$, and temperature as well as EEG were monitored continuously after paralysis, and the level of anesthetic was increased when necessary. Pupils were dilated with atropine eye drops, and clear gas permeable contact lenses were used to render the retina conjugated with the stimulus monitor 28.5 or $57 \mathrm{~cm}$ distant. In most animals, $3 \mathrm{~mm}$ artificial pupils were used. The optic disks and areae centralii were plotted on the screen.

A craniotomy was made over V1, V2, and V3 and surrounding visual areas, and the dura was reflected. An optical imaging chamber was cemented to the skull and filled with artificial CSF using the following composition (in mM): $118 \mathrm{NaCl}, 4.8 \mathrm{KCl}, 2.5 \mathrm{CaCl}_{2}, 25 \mathrm{NaHCO}_{3}, 1.2$ $\mathrm{MgSO}_{4}, 1.2 \mathrm{KH}_{2} \mathrm{PO}_{4}, 10$ glucose; and sealed with a coverslip glass. In some cases, openings were simply sealed with $1 \%$ agarose under a glass coverslip without a chamber.

Visual stimuli and optical imaging. Intrinsic optical imaging signals were acquired with the Imager 2001 differential video-enhancement imaging system and data acquisition software (Optical Imaging, Mountainside, NJ). Surface reference images of cortical vasculature were acquired with a $540 \mathrm{~nm}$ green light. The cortex was illuminated with a $611 \mathrm{~nm}$ orange light during data acquisition and was visualized with a tandem lens macroscope attached to a low-noise video camera. The camera was focused slightly beneath the cortical surface, and the depth of field was subsequently increased by closing the lens diaphragm. Visual stimuli were generated using a visual stimulus generator system (Cambridge Research Systems, Rochester, UK) and were presented on a 21 inch video screen (Sony FD Trinitron, model GDM-F400; Sony, Tokyo, Japan) in $120 \mathrm{~Hz}$ noninterlaced mode with a mean luminance of $30 \mathrm{~cd} / \mathrm{m}^{2}$.

To study the functional organization of orientation preference, highcontrast rectangular gratings (fundamental spatial frequency, 0.5 cycles/ degree; drift velocity, $2 \mathrm{~Hz}$; contrast, $100 \%$; duty cycle, 20\%) of four or eight orientations were displayed on the full screen and presented binocularly. Each grating was moved back and forth along an axis that was orthogonal to the orientation of the grating. A single trial consisted of data acquisition during continued presentation of the drifting grating stimulus for 8 sec and an interstimulus interval of $10-12$ sec using a blank screen of mean luminance. Stimulus sets were made up of 10-30 trials. In some trials, all orientation conditions and a blank control were presented in a randomized order. In other trials, only orthogonal pairs of orientations (e.g., $0 / 90^{\circ}, 45 / 135^{\circ}$ ) were presented in separate trials.

For visuotopic optical image mapping, spatially limited horizontal and vertical grating stimuli were presented monocularly either within $1-4^{\circ}$ rectangular windows or $2-4^{\circ}$ patches at eccentricities ranging from 0 to $20^{\circ}$ (Lyon et al., 2002). Given the limitations of our camera view, we imaged only the dorsal cortex, which represents the lower visual field in all three visual areas $(\mathrm{V} 1, \mathrm{~V} 2, \mathrm{~V} 3)$ at eccentricities ranging from 0 to $20^{\circ}$. Borders of visual areas were determined by comparing $\mathrm{CO}$ staining patterns with visuotopic maps produced by optical imaging.

Video images were acquired at a rate of 30 frames/sec, but all frames acquired for each condition during the $8 \mathrm{sec}$ period were summed together into four to eight data frames before additional analysis. Individual data frames included $744 \times 480$ pixels, with a resolution of either 87 pixels/mm for the $50 \mathrm{~mm}$ top, $50 \mathrm{~mm}$ bottom lens combination, or 174 pixels $/ \mathrm{mm}$ for the same lens combination used with the addition of a $2 \times$ converter.

Image analysis and measurement. To assess the intrinsic signal strength, we measured the intensity of optical reflectance during the stimulus condition relative to the intensity during the blank control $(\Delta R / R)$. Specifically, the signal time courses were examined by plotting the pixel values from the two blank control frames (frames 1,2) and the subsequent eight data frames (frames 3-10) associated with $8 \mathrm{sec}$ stimulation period. Because the values from frames 7 and 8 exhibited peak reflectance changes, the $\Delta R / R$ was calculated as: ([average $(\mathrm{F} 7+\mathrm{F} 8)-\mathrm{F} 1] / \mathrm{F} 1] \times 100 \%)$. We averaged $\Delta R / R$ across map pixels of specific domains of four different orientations from 10 different trials.

To create visuotopic maps, all images associated with stimuli of the same position were summed and divided by the pure blank" obtained by summing the images of the blank control to create single-condition maps. The resulting maps were clipped at 1-2 SDs from the mean of the image pixel distribution, smoothed using a $40 \mu \mathrm{m}$ radius mean filter kernel, and scaled in the range of 0-255 gray levels for appropriate display.

To construct maps of orientation preference, we summed together all images associated with the same orientation using Winmix software (Optical Imaging); the summed images acquired during the presentation of one orientation were divided by the summed images acquired during presentation of the orthogonal orientation to create orientation difference maps (Blasdel, 1992a; Bosking et al., 1997). These difference images were "clipped" at 1-2 SDs around the mean of the image pixel distribution and scaled in the range of 0-255 gray levels.

Custom programs written in NIH Image (as an extension to the public domain National Institutes of Health Image program, at http://rsb.info.nih.gov/nih-image/) and Matlab (MathWorks, Natick, MA) were used to further process the data. Orientation difference images were smoothed using a $40 \mu \mathrm{m}$ radius mean filter kernel. Low frequency noise was reduced by convolving the image with a $450 \mu \mathrm{m}$ radius mean filter kernel and subtracting the result from the original image (Bosking et al., 1997).

Iso-orientation domain sizes were measured on orientation difference maps, in which dark areas indicate regions that responded strongly to one particular orientation, and light areas responded strongly to the orthogonal orientation. For all four difference maps, the same region of interest (ROI) was chosen, which included at least 20 orientation domains that were completely contained within the ROI. Each ROI of each image was thresholded at the top 25\% darkest pixel level to include only dark domains. The Imaging Processing Toolkit (Reindeer Graphic Company, Raleigh, NC) was used to automatically measure these domain sizes. The average iso-orientation domain size from each case was based on the measurements of four individual orientations $\left(0,45,90\right.$, and $\left.135^{\circ}\right)$.

Vector summation of the difference images was done on a pixel by pixel basis to create a color-coded orientation preference (angle) map and a magnitude (vector strength) map coding the degree of orientation selectivity by brightness, with strong orientation selectivity represented as the brightest, and weak orientation selectivity as the darkest (Bosking et al., 1997). The two maps were combined to create a polar map, which contained information about both orientation preference (color) and magnitude of orientation selectivity (brightness). Maps showing rate of change of orientation preference were also constructed for the same regions of cortex with the highest rate of change of orientation represented as white and the lowest as black. Locations of potential pinwheel centers were determined by thresholding the rate of change map to highlight those areas where the rate of change was greatest. Before assigning these sites as pinwheel centers, however, we checked the original angle maps to ensure that all orientations were represented around these points and only quantified regions that were relatively free of blood vessel artifacts. 

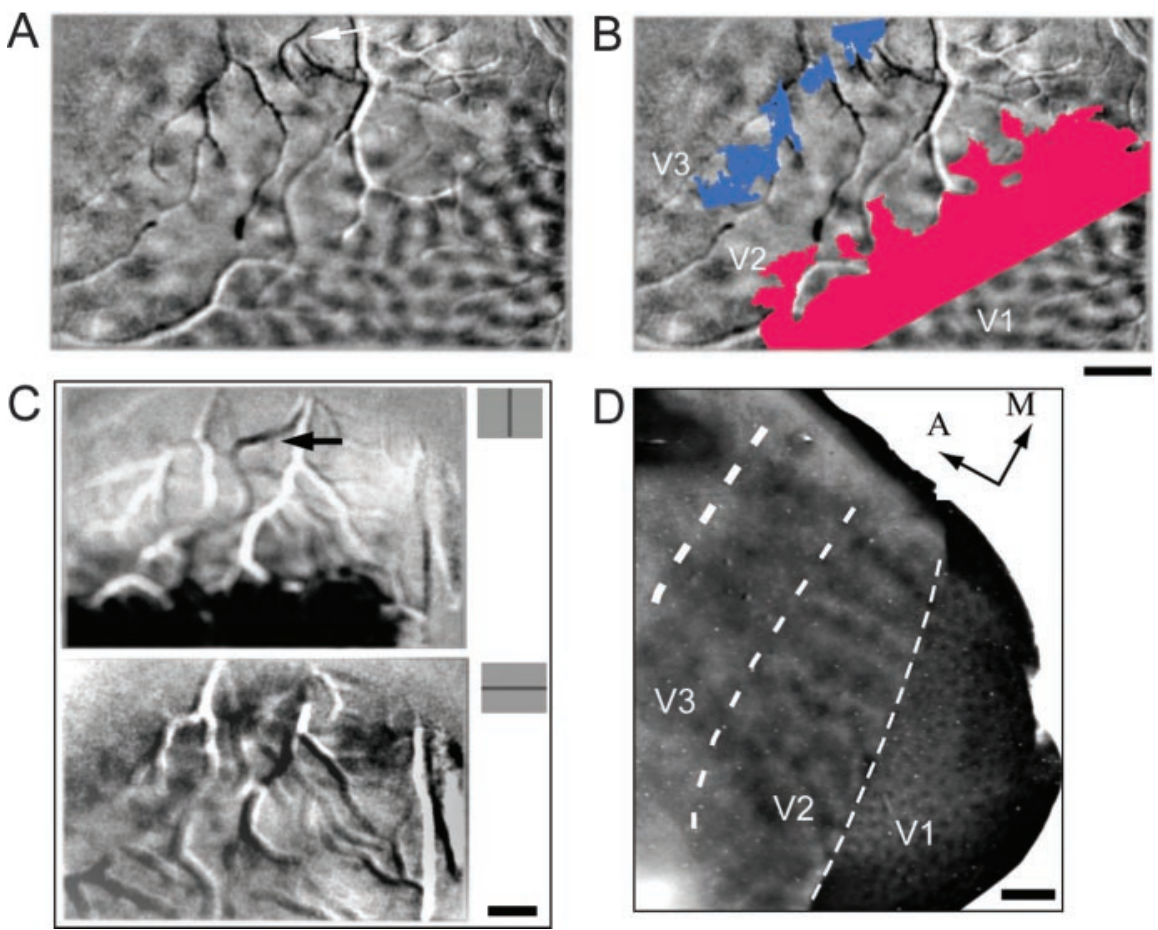

Figure 1. Visuotopic organization of V1, V2, and V3. A shows an orientation difference map of $0 / 90^{\circ}$ for $\mathrm{V} 1, \mathrm{~V} 2$, and $\mathrm{V} 3$, where $0^{\circ}$ activation is shown as dark patches and $90^{\circ}$ as light patches. $B$ is the orientation map shown in $A$ superimposed on the visuotopic map taken from C (the vertical meridian activation is shown in red, and the horizontal meridian activation is shown in blue). $C$ shows single condition maps for cortical activation patterns resulting from stimulation of $2^{\circ}$ stimuli presented separately along the vertical meridian (top panel) and the horizontal meridian (bottom panel) (see the icons). The white arrow in $A$ and the black arrow in the top panel in ( point to the common blood vessel landmark used for alignment of the two images. Because of camera angle limitations, we imaged only the dorsal portion of visual cortex (corresponding to the representation of the lower visual field). $D$ shows a CO section with V1, V2, and V3 delineated by thin and thick dashed lines from a different case. The modules in V1 are blob-like, those in V2 are stripe-like, and those in V3 are larger, patch-like, and more irregular than in V2. A, Anterior; M, medial. Scale bars: $A-C, 1 \mathrm{~mm} ; D, 2 \mathrm{~mm}$.

Because it has been argued that light scatter may distort images in a way that could prevent accurate determination of pinwheel centers (Granquist-Fraser et al., 2003; Schwartz et al., 2003), we focused either on the cortical surface or very near the surface.

In some cases, to reduce vascular artifacts, we used reference images or a stack of optical images to create a mask indicating the location of the major blood vessels. The grayscale value for each pixel in the data images that was located in the mask was replaced by the mean of the grayscale values of the appropriate surrounding pixels outside the mask (Bosking et al., 2000; Blasdel and Campbell, 2001). Grayscale values for those pixels that were not in the mask were not changed during this filtering. Quantitative measures were always made in regions outside of the masked areas.

Electrophysiological verification. After optical imaging sessions, multiunit or single-unit electrode recordings were made to test cell responses within regions of specific orientations defined by the imaging. Cells were recorded in the superficial cortical layers with Parylene-coated tungsten electrodes of 1-2.5 M $\Omega$ impedance (Micro Probe, Potomac, MD). Recordings of orientation preference were consistent with the optical imaging results.

Histology, alignment, and data analysis. At the termination of each experiment, the monkey was deeply anesthetized with an overdose of sodium pentobarbital and perfused transcardially with a saline rinse followed by $2 \%$ paraformaldehyde in $0.1 \mathrm{~m}$ phosphate buffer. The brain was removed, and the imaged area of cortex was separated and flattened. The imaged piece of cortex was then frozen and cut with the surface vascular pattern preserved in the first 100-150 $\mu \mathrm{m}$ section. Subsequent sections were cut at $50 \mu \mathrm{m}$. CO staining was performed using methods described previously (Boyd and Matsubara, 1996).

Surface and radial blood vessels were the primary landmarks used to align histological sections to the reference images. Differences between images and sections resulting from distortion or tissue shrinkage ( 10-15\%) were handled by global scaling and rotation. After the optical images were aligned with the histological data, ROIs were chosen for additional quantification. These regions were selected because they contained dense and even $\mathrm{CO}$ staining and could be aligned with optical images that were relatively free of blood vessel artifacts. Comparisons between cases were always made at the same relative visual field location (eccentricity).

In V1, the CO image was high-pass filtered at slightly larger than the average blob size (at a radius of $70-170 \mu \mathrm{m}$ ) and low-pass filtered at slightly smaller than the smallest blobs (at a radius of 35-70 $\mu \mathrm{m}$ ). This image was then thresholded and posterized into three gray levels: dark (CO blob proper), white (CO interblob proper), and gray (border area) (see Fig. 4B) (Boyd and Casagrande, 1999). Using Igor Pro 4.0 (WaveMetrics, Lake Oswego, OR), we encoded the X-Y coordinates of pinwheel centers (defined as described above) and then transferred these coordinates to the CO thresholded image to examine the relationship between pinwheel centers and blob, interblob, and blobinterblob border areas. Next, $\chi^{2}$ analyses was performed to assess the probability of pinwheel centers falling within each of these compartments. Additionally, to compare the degree of orientation selectivity between compartments, we performed a statistical analysis (KruskalWallis test) on the relationship between $\mathrm{CO}$ modules (CO blobs and interblobs) and orientation selectivity. Response selectivity was calculated by dividing the resultant vector strength for each pixel by the sum of response activities of four stimulus orientations (Galuske et al., 2002). This orientation selectivity index ranged from 0 to 1 (lowest to highest).

The relationship between orientation selectivity and CO intensity was analyzed in a similar manner in areas V2 and V3. CO images were appropriately bandpass filtered (high-pass at a radius of $340-680 \mu \mathrm{m}$; lowpass at a radius of 100-200 $\mu \mathrm{m}$ ) and thresholded into dark CO bands, interbands, and border areas. We then transferred contour plots of CO maps in V2 and V3 to the optical images to qualitatively and quantitatively examine the correspondence of orientation selectivity with different $\mathrm{CO}$ compartments.

A one-way ANOVA with post hoc mean difference tests (Tukey tests) was performed, provided that the data did not violate the prerequisite of variance homogeneity across groups. For groups with both unequal variances and unequal samples, we used the Kruskal-Wallis test and the Mann-Whitney $U$ test for group comparisons. Alpha levels of $p \leq 0.05$ were considered significant.

\section{Results}

Our most significant finding was that the modular functional organization of V1 and V2 of owl monkeys was strikingly similar to that described in other simian primates, suggesting that differences in lifestyle (nocturnal versus diurnal), including the presence or absence of color vision, is not a driving force behind the existence of these functional modules. We also demonstrated for the first time that V3, like V2, consists of zones of high and low orientation selectivity that are oriented perpendicular to the $\mathrm{V} 2 / \mathrm{V} 3$ border. In all three areas, the basic geometric arrangement in regions of high orientation selectivity was similar and consisted 
of pinwheels and linear zones, although the size of individual orientation domains increased significantly from V1 to V2 to V3. Below, we consider these results in more detail.

\section{Defining visual areas}

We defined visual areas on the basis of a combination of visuotopic mapping and functional imaging, histological landmarks, and predictions concerning the extent of each area determined by connectional anatomy (Lyon and Kaas, 2001, 2002; Lyon et al., 2002). Figure $1 A$ shows that there are clear differences in orientation preference organization between V1, V2, and V3 (see details below). Figure 1C shows cortical activation patterns resulting from stimulation of the vertical meridian (V1/V2 border) and the horizontal meridian (V2/V3 border), and Figure $1 B$ shows the overlay of these activation patterns (vertical meridian activation in red; horizontal meridian in blue) on the orientation map as shown in Figure $1 \mathrm{~A}$. Consistent with the known visuotopies of these areas, restricted stimuli presented along the vertical meridian activated cortex on the V1/V2 border as well as along the anterior border for V3; restricted stimuli presented along the horizontal meridian crossed through V1 and activated cortical tissue marking the border of V2 and V3 (Lyon et al., 2002). In CO-stained sections, V1 was easily distinguished from $\mathrm{V} 2$ on the basis of the presence of CO blobs and the absence of stripe-like CO patterns seen in V2. Similarly, V3 could be distinguished from V2 by its much larger light and dark patches (Fig. 1D). Areal borders determined by visutopic mapping with optical imaging were consistent with the borders determined by CO staining.

\section{The general organization of orientation preference in V1, V2, and V3}

Figure 2 shows four orientation difference maps in V1 and V2. The borders of $\mathrm{V} 1 / \mathrm{V} 2$ and $\mathrm{V} 2 / \mathrm{V} 3$, indicated by thin and thick dashed lines in these images, respectively, were determined on the basis of the criteria described above and in Materials and Methods. In these orientation difference maps, dark areas indicate areas that responded strongly to a particular orientation, and light areas indicate areas that responded strongly to the orthogonal orientation. In both V1 and V2, visual stimuli of different orientations elicited clear patches of orientation-specific activity. As illustrated in Figure 2, there were marked differences in the organization of orientation preference domains between V1 and V2. For example, the size and spacing of iso-orientation domains in V2 appeared to be approximately twice as large as those found in V1. Furthermore, whereas iso-orientation domains in V1 formed a continuous map of high orientation selectivity, in V2, clear bands of high and low orientation selectivity running perpendicular to the V1/V2 border were evident that resembled the pattern of high and low CO-stained bands in this area. This organization of orientation selectivity in V2 is strikingly different from that demonstrated in another nocturnal primate (bush baby) where the organization of V2 shows no bands of low and high orientation selectivity and resembles the organization of V1 (Bosking et al., 1996) but is similar to that described in diurnal primates such as macaque monkeys and squirrel monkeys (Ts'o et al., 1990; Malach et al., 1994; Roe and Ts'o, 1995).

Figure 3 shows the maps of orientation preference in V1 and V2 in more detail. Figure $3 A, B$, and $C$, show an orientation preference map, a magnitude map, and a polar map, respectively, for the same case shown in Figure 2. As reported in other primate and nonprimate species (Blasdel, 1992b; Chapman et al., 1996; Bosking et al., 1997; Shmuel and Grinvald, 2000), the owl monkey V1 map contained regions where orientation preference changed linearly (linear zones), and regions where orientation preference was organized radially (pinwheels) (Figs. 3A,C-E, $4 B$ ). Linear zones were often prominent along the V1/V2 border, and iso-orientation contour lines in these linear zones tended to intersect the border at right angles (Fig. 3E). The average size of iso-orientation domains in V1 $(n=5$ cases $)$ was $0.076 \pm 0.046$ $\mathrm{mm}^{2}$ (mean $\pm \mathrm{SD}$ ).

V2 bands of high and low orientation selectivity, which were evident under both low (Fig. $3 B, C, E$ ) and high (Fig. 3D) magnification, were present in all 10 cases studied. Nevertheless, the regularity of these bands varied somewhat between cases (Fig. 3, compare $E$ with $C$ and $D$ ). In many cases, as also described in macaque monkey (Roe and Ts'o, 1995), the V2 bands in owl monkey had irregular shapes suggestive of internal substructure. A similar irregular patchy appearance often was seen in the CO stained bands as well (see below) (Tootell et al., 1985). Average widths of bands of high and low orientation selectivity in V2 measured in four cases parallel to the V1/V2 border were $1.7 \pm$ $0.5 \mathrm{~mm}$ (mean $\pm \mathrm{SD}$ ) and $1.0 \pm 0.3 \mathrm{~mm}$, respectively, which differed significantly ( $p<0.0007$; $t$ test).

Quantitative analysis matched our qualitative impression that the size of $\mathrm{V} 2$ iso-orientation domains $\left(0.10 \pm 0.034 \mathrm{~mm}^{2}\right)$ was 

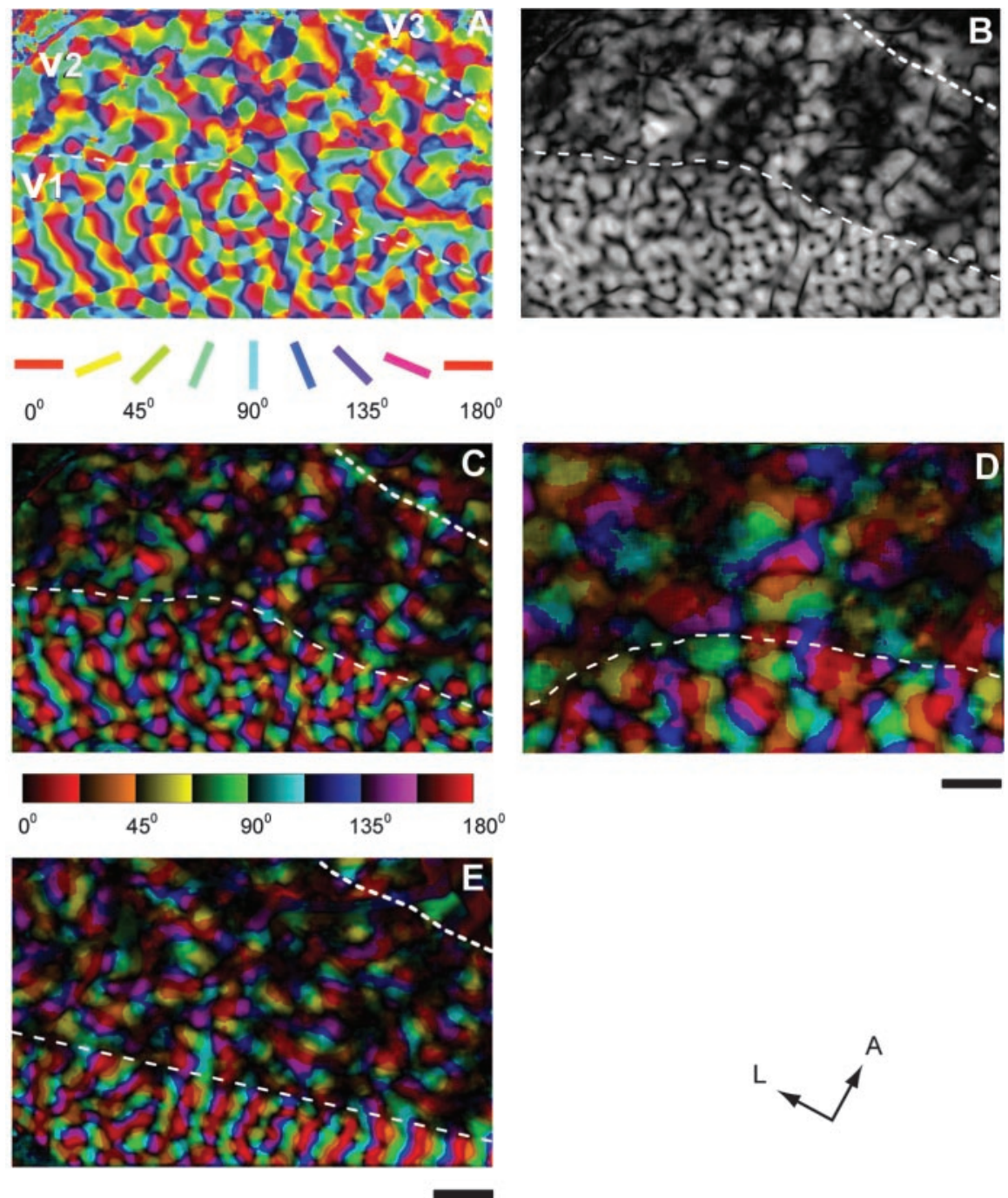

Figure 3. Functional maps of orientation preference in V1 and V2. $A, B$, and C show an orientation preference map, a magnitude map, and a polar map, respectively, produced from vector summation on a pixel by pixel basis from the difference images shown in the case pictured in Figure 1. The orientation preference map $(A)$ is color-coded according to the key shown below the map. The magnitude map $(B)$ shows the overall strength of orientation selectivity (light, more selective; dark, less selective). The polar map $(C)$ contains information about both orientation preference (color) and magnitude of orientation selectivity (brightness) (see the key below). $D$ is a polar map from a different case obtained with a higher-power lens. E shows another polar map from a third case in which regions of high and low orientation selectivity in V2 are not as regular and stripe-like as those in $C$ and $D$. The thin and thick dashed lines delineate the V1/V2 and V2/V3 borders, respectively. A, Anterior; L, lateral. Scale bars: (in $E) A-C$, $E, 1 \mathrm{~mm} ; D, 0.5 \mathrm{~mm}$. See Results for details.

significantly larger than those of V1 ( $p<0.0005$; $t$ test). However, the basic geometric arrangement of the map of orientation preference (within regions of high selectivity) was quite similar across V1, V2, and V3, with all three visual areas showing both pinwheels and linear zones (Figs. 3, 4).

Because a low magnitude of orientation selectivity can result from the vector summation procedure, either because of low orientation selectivity or because of low absolute levels of response in some areas, we performed an additional analysis to test whether absolute levels of response were lower in the V2 regions with low selectivity. We measured the intrinsic signal amplitudes, $\Delta R / R$ (the intensity of optical reflectance during the stimulus condition relative to the intensity during the blank control) in $\mathrm{V} 1$ as well as bands of high and low orientation selectivity in V2. For the case shown in Figure $3 D$, the amplitudes $(\Delta R / R)$ measured from four orientations ranged from -0.236 to $-0.416 \%$ in $\mathrm{V} 1$ (mean, $-0.316 \pm 0.02 \%$; mean $\pm \mathrm{SE}$ ); for areas of high orientation selectivity in V2, it ranged from -0.115 to $-0.157 \%$ (mean, $-0.144 \pm 0.007 \%$ ); for areas of low orientation selectivity in V2, it was from -0.074 tk;4to $-0.167 \%$ (mean, $-0.115 \pm 0.01 \%$ ). Although the amplitudes differed significantly between $\mathrm{V} 1$ and $\mathrm{V} 2$, the $\mathrm{V} 2$ regions of low and high orientation selectivity showed a comparable response range $(n=$ 2 cases). Thus, the regions of low orientation selectivity did not appear to be associated with low responsivity.

$\mathrm{V} 3$ was similar to V2 and distinct from $\mathrm{V} 1$ in that it contained zones of high and low orientation selectivity that ran perpendicular to the V2/V3 border. V3 was distinguished from both $\mathrm{V} 1$ and $\mathrm{V} 2$ by its significantly larger iso-orientation domains (Fig. 4A). The average iso-orientation domain size for $\mathrm{V} 3$ measured in three cases was $0.126 \pm 0.055 \mathrm{~mm}^{2}$. One-way ANOVA and post hoc mean difference tests indicated that iso-orientation domain sizes in V1, V2, and V3 differed significantly (one-way ANOVA; $p<0.0001$; Tukey tests; V1 vs V3, $p<0.001$; V2 vs V3, $p=0.006)$. These differences were also reflected in the differences in pinwheel density between areas. Overall, pinwheels occurred at a density of $7.7 \pm 1.2$ pinwheels $/ \mathrm{mm}^{2}$ (mean $\pm \mathrm{SD}$ ) in V1 (for $0-12^{\circ}$ of eccentricity), $3.04 \pm 0.55$ pinwheels $/ \mathrm{mm}^{2}$ in $\mathrm{V} 2$, and $2.36 \pm 0.1$ pinwheels $/ \mathrm{mm}^{2}$ in V3.

Although V3, like V2, exhibited zones of high and low orientation selectivity, the $\mathrm{V} 3$ zones were not simple continuations of the V2 bands of high and low orientation selectivity. Instead, as shown in Figure 4, V2 bands of high orientation selectivity appeared to fuse into larger zones of high orientation selectivity in V3. Some V2 bands of low orientation selectivity stopped abruptly at the V2/V3 border, and other V2 bands of low orientation selectivity continued uninterrupted across the V2/V3 border (Fig. 4, single and double arrows). The average widths of regions of high and low orientation selectivity in V3 measured parallel to the V2/V3 border from two cases, in which these zones were particularly well defined, were $2.8 \pm 0.6 \mathrm{~mm}$ (mean $\pm \mathrm{SD}$ ) and $1.3 \pm 0.2 \mathrm{~mm}$, respectively. Thus, the high orientation selectivity zones in V3 were, on average, approximately twice the size of those found in V2, whereas the low orientation selectivity V3 zones were on average approximately the same as those in V2.

\section{Relationship between pinwheel centers and CO blobs in V1}

We examined the relationship between the map of orientation preference and the distribution of CO blobs. Figure 5, $A$ and $B$, shows an example of the precision of alignment between a $\mathrm{CO}$ stained (Fig. 5A) section and the optical image (Fig. 5B). The CO image was then thresholded and posterized into three gray levels: dark (CO blob proper), white (CO interblob proper), and gray 
(border area) (Fig. 5D,E). The pinwheel centers marked as black dots in the orientation preference map shown in Figure 5C were transferred to the CO threshold image (Fig. $5 F$ ) to examine the distribution over CO blob, interblob, and border areas. The average density of pinwheel centers in CO blobs was $8.65 / \mathrm{mm}^{2}$, in interblobs it was $7.4 / \mathrm{mm}^{2}$, and in border areas between blobs and interblobs it was $7.95 / \mathrm{mm}^{2}$ (Fig. $5 F)$. A $\chi^{2}$ analysis indicated that there was no significant relationship in the distribution of pinwheel centers to CO blobs, interblobs, or border areas ( $p=0.54)$. Similar results were obtained from three other cases at eccentricities of $\leq 12^{\circ}$ (Table 1 ). These results, indicating the lack of a relationship between the map of orientation selectivity and CO blobs, are consistent with two previous studies that also examined relationships between pinwheel centers and CO blobs in primate V1 (macaque: Bartfeld and Grinvald, 1992; bush baby: Bosking et al., 1996).

We also performed a statistical analysis on the relationship between $\mathrm{CO}$ modules and orientation response selectivity. The orientation selectivity indices (see Materials and Methods) for pixels aligned with CO blobs did not differ significantly from those aligned with interblobs ( $n=4$ cases). In the case shown in Figure 5, the average orientation selectivity index was $0.70 \pm 0.41$ for blobs, $0.78 \pm 0.46$ for interblobs, and $0.69 \pm 0.41$ for border areas ( $p>0.05$; one-way ANOVA).

\section{Relationship of orientation preference domains to the $\mathrm{CO}$ bands in V2 and V3}

Based on qualitative analyses, V2 CO thin bands in macaque monkeys and squirrel monkeys have been reported to correspond to zones of low orientation selectivity and high chromatic selectivity, whereas V2 CO thick bands have been reported to correspond to zones of high orientation selectivity (DeYoe and Van Essen, 1985; Ts'o et al., 1990; Malach et al., 1994; Roe and Ts'o, 1999; Xiao et al., 2003). Areas V2 and V3 of owl monkeys are also characterized by bands of light and dark CO staining (Tootell et al., 1985; Lyon and Kaas, 2002) (Figs. 6, 7). These bands appeared to correspond qualitatively to zones of high and low orientation selectivity. To facilitate quantitative comparisons, we divided V2 (as we had done in V1 for CO blobs) into dark CO bands, interbands, and border areas with appropriate thresholding of CO images (see Materials and Methods) (Fig. 6). We also subdivided CO dark bands into two types: CO thick bands and $\mathrm{CO}$ thin bands, based on the assumption that if two functional compartments exist, they should alternate in sets to map all functional compartments efficiently without "holes" at each eccentricity. It is noteworthy that quantifying these relationships was more complex in V2 because of, in part, the fact that each "band" was not uniform either in CO staining or in its degree of orientation selectivity. Rather, these bands often appeared more like a string of smaller patches. The results of our analyses are illustrated in Figure 6, where the dark areas (low selectivity) in the polar map were centered on the $\mathrm{CO}$ dark patches in the thin bands, and the bright areas (high selectivity) in the magnitude and polar maps (Fig. 6C,D,F) were centered principally on $\mathrm{CO}$ dark patches of the thick bands. We performed statistical analysis on the relationship between V2 CO modules (CO thick bands, $\mathrm{CO}$ thin bands, and pale bands) and orientation response selectivity in the V2 map. In Figure $6 C$, the orientation selectivity indices for map pixels over dark patches of $\mathrm{CO}$ thick bands were significantly higher than those over $\mathrm{CO}$ dark patches in thin bands (thick CO dark patches: $0.32 \pm 0.16$; mean $\pm \mathrm{SD}$; thin $\mathrm{CO}$ dark patches: $0.18 \pm 0.13$; pale patches: $0.29 \pm 0.16$; border areas: $0.26 \pm 0.17 ; p<0.0001$; Kruskal-Wallis test). These same results were found in three other cases examined, suggesting that $\mathrm{CO}$ thick and thin dark bands represent distinct functional compartments.

As Malach et al. (1994) observed in squirrel monkey V2, we also noted that the map of orientation preference did not appear to be constrained by the borders between $\mathrm{CO}$ thick, thin, and pale bands but bridged the transition between these sets of bands. In owl monkeys, some pale bands exhibited high orientation selectivity, whereas other pale bands tended to exhibit low orientation selectivity (Fig. 6C, D, F). Statistical analysis confirmed our qualitative impression that two types of pale bands exist, which we refer to here as type I and type II. Type I CO pale bands lie lateral to CO thick bands, and type II CO pale bands lie lateral to thin CO bands. In Figure 6C, the orientation selectivity indices for V2 map pixels over the type II CO pale bands differed significantly from those over the type I CO pale bands (type II CO pale bands: $0.31 \pm 0.17$; type I CO pale bands: $0.21 \pm 0.13$; $p<0.001$; Kruskal-Wallis test). The pattern of bands appeared to alternate as follows: a thin CO dark band of low orientation selectivity, a pale band of low orientation selectivity (type I), a thick CO dark band of high orientation selectivity, a pale band of high orientation selectivity (type II). This pattern alternated at least once in a single V2 image, and the trend was seen consistently in four ani- 

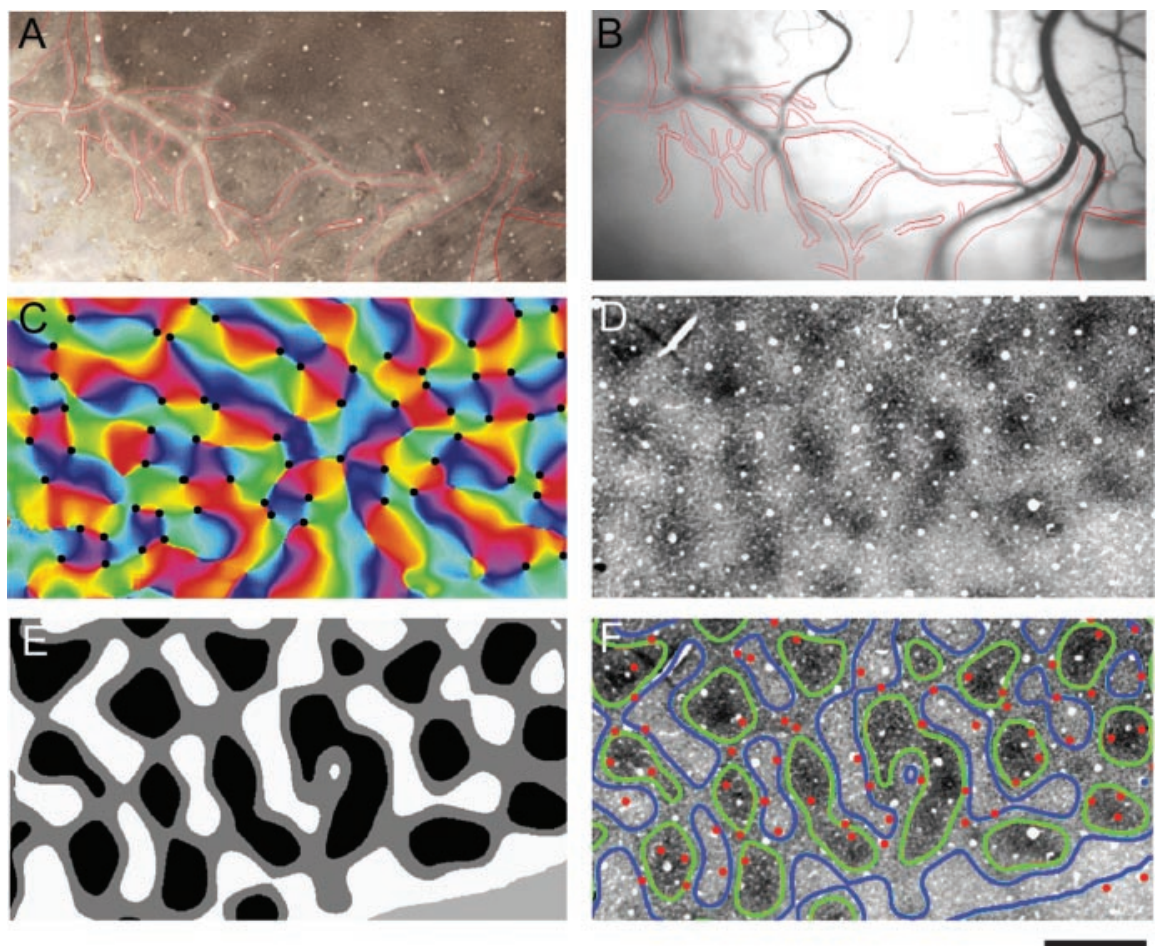

Figure 5. The relationship between pinwheels, CO blobs, and interblobs in V1. A shows a histological section with superficial blood vessel contours traced in red. $B$ is the optical reference image with the overlay of the superficial blood vessel patterns (red contours) shown in $A$. ( is an orientation preference map with pinwheel centers identified (black dots) from the imaged area. $D$ is a $C 0$ section from the imaged area. $E$ is taken from a $\mathrm{CO}$ section in $D$ in which the image was thresholded into three zones on the basis of $\mathrm{CO}$ density, dark $\mathrm{CO}$-blob centers, gray $\mathrm{CO}$-blob border regions, and light $\mathrm{CO}$-interblobs. $\mathrm{F}$ is the $\mathrm{CO}$ section with contour plots transferred from $E$ with pinwheel center locations from Cindicated (red dots). Green contours encircle $C O$ blobs, blue contours encompass $\mathrm{C} 0$ interblobs, border areas lie between the two colors. In the area analyzed, the pinwheel density was $8.65 / \mathrm{mm}^{2}$ for CO blobs, $7.4 / \mathrm{mm}^{2}$ for interblobs, and $7.95 / \mathrm{mm}^{2}$ for border areas. The distributions do not differ significantly ( $p=0.54 ; \chi^{2}$ analysis). Scale bar, $1 \mathrm{~mm}$.

mals in either left or right hemisphere. From these data, one would conclude that there are actually at least four functional compartments in V2, two associated with $\mathrm{CO}$ dark bands and two associated with $\mathrm{CO}$ pale bands.

In V3, the large CO dense zones always appeared to correspond to zones of high orientation selectivity, whereas the $\mathrm{CO}$ pale zones in V3 correlated with regions of low orientation selectivity (Fig. $7 A-D$ ). We applied a similar quantitative analysis to V3 that we described above for V2. In V3, we compared the orientation selectivity indices for regions of the map that corresponded to $\mathrm{CO}$ dense and $\mathrm{CO}$ pale zones. The magnitude of orientation selectivity over the $\mathrm{CO}$ dense zones was significantly greater than the magnitude of orientation selectivity over CO pale zones for two cases that were measured (case 1: V3 CO dense zones, $0.21 \pm 0.24 ; \mathrm{V} 3 \mathrm{CO}$ pale zones, $0.12 \pm 0.20$; case 2 : CO dense zones, $0.35 \pm 0.19$; CO pale zones, $0.20 \pm 0.14$; both cases, $p<0.005$; Kruskal-Wallis test). Thus, CO dense zones in V3 correlate functionally with high orientation selectivity, whereas $\mathrm{CO}$ pale zones correlate with regions of low orientation selectivity.

\section{Discussion}

In this study, we had two goals: (1) to determine whether the functional organization of V1, V2, and V3 in owl monkeys differs from that observed in other primates, and (2) to quantify the geometry of orientation domains in relationship to compartments defined by $\mathrm{CO}$ in these three areas. Our results suggest that most, but not all, elements of the functional organization of areas
$\mathrm{V} 1, \mathrm{~V} 2$, and V3 are shared across primate species. Differences in the organization of V2 that have been observed appear to relate more to evolutionary relationships than to nocturnal versus diurnal lifestyle. Our quantitative results in owl monkeys suggest that in V1, there is no specific spatial relationship between the map of orientation selectivity and CO blobs, although a consistent numerical relationship of two pinwheels for every one blob was observed. In V2, the relationship between $\mathrm{CO}$ and orientation selectivity provides evidence for at least four functional domains, whereas in V3, we find evidence for two functional compartments. Below, we consider the implications of these findings in light of results reported by others.

\section{CO blobs and orientation preference in $\mathrm{V} 1$}

Livingstone and Hubel $(1984,1988)$ proposed that CO blobs in V1 contain a high proportion of nonoriented, color-selective cells. It has been clear, however, for some time that these domains do not exist solely for the purpose of segregating colorselective cells in all species, because all primates studied have CO blobs, even those that are nocturnal and those that lack color vision, such as the owl monkey (Casagrande and Kaas, 1994). Whether these CO blobs in some diurnal simians contain a high proportion of nonoriented, colorselective cells as originally proposed remains controversial. Electrophysiological support for this type of segregation has usually been obtained by subjective classification of single-cell recordings into one of a number of categories (Livingstone and Hubel, 1984; Ts'o and Gilbert, 1988). Furthermore, some electrophysiological investigations have reached the opposite conclusion (Lennie et al., 1990; Edwards et al., 1995; Leventhal et al., 1995). Recent optical imaging experiments have provided strong qualitative support for the clustering of color-selective cells in blobs, although even in this study, color-selective patches were never confined to CO blobs (Landisman and Ts'o, 2002a). However, similar support for the lack of orientation selectivity in blobs has not been obtained. Instead, optical imaging experiments in the macaque monkeys have consistently found that the only areas of low orientation selectivity in V1 are near pinwheel centers, and that the pinwheel centers are not specifically aligned with blobs (Bartfeld and Grinvald, 1992). In the owl monkey, there is no significant relationship between CO blobs-interblobs and orientation selectivity, in agreement with studies of single-unit recordings in V1, which showed little difference in the orientation selectivity of individual neurons in blobs and interblobs (O'Keefe et al., 1998). A lack of relationship between orientation selectivity and CO blobs has also been observed in the bush baby (DeBruyn et al., 1993; Bosking et al., 1996) and thus may be a common feature across all primates.

Although we found no specific spatial relationship between the map of orientation selectivity and the blobs, we observed a consistent 2:1 relationship in the number of pinwheels relative to 
Table 1. The relationship between pinwheel centers and $\mathrm{CO}$ blobs

\begin{tabular}{|c|c|c|c|c|}
\hline \multirow[b]{2}{*}{ Case number } & \multicolumn{3}{|c|}{ Orientation pinwheel center density (per $\mathrm{mm}^{2}$ ) } & \multirow[b]{2}{*}{$\chi^{2}$ analysis $p$ value } \\
\hline & Within CO blobs & Within CO interblobs & Within border areas & \\
\hline 01.15 .02 & 8.68 & 7.40 & 7.96 & 0.54 \\
\hline 11.07 .01 & 5.60 & 5.47 & 7.83 & 0.38 \\
\hline 12.06 .01 & 6.78 & 6.40 & 7.40 & 0.68 \\
\hline 11.01 .01 & 7.93 & 8.05 & 7.27 & 0.78 \\
\hline 02.26 .02 & 8.54 & 5.12 & 4.02 & 0.07 \\
\hline
\end{tabular}

The $\mathrm{V} 1$ areas selected for analysis ranged from 5 to $12 \mathrm{~mm}^{2}$, with eccentricities $\leq 12^{\circ}$. Alpha levels of $p \leq 0.05$ are considered significant.

the number of blobs. This ratio has been found in several species, as shown in Table 2. The structure of the map of orientation preference is also very similar across a wide range of species, including owl monkeys, macaque monkeys, squirrel monkeys, bush babies, ferrets, tree shrews, and cats (Blasdel, 1992a,b; Malach et al., 1994; Chapman et al., 1996; Bosking et al., 1997; Shmuel and Grinvald, 2000; our study). These consistencies in the organization of the map of orientation preference and the periodicity of this map relative to the periodicity of the CO blobs may reflect a compromise between the need to represent all feature combinations for every location in visual space (coverage) and the need to maintain similarity between neighboring columns of cells (continuity) (Swindale et al., 2000).

Given that lower spatial frequencies appear to be preferentially associated with CO blobs in bush babies, owl monkeys, and even in nonprimates (i.e., cats) (Shoham et al., 1997; Xu et al., 2003a,b), the pinwheels located in blobs may be related to low spatial frequency processing (Issa et al., 2000), which is consistent with the speculation that properties that require relatively constant metabolic expenditure such as color contrast, brightness contrast, and other surface properties are represented separately in CO blobs (Allman and Zucker, 1990).

\section{The organization of orientation and CO compartments in V2} The original hypothesis concerning CO stripes in V2 was that they identified three functional compartments related to the processing of color (thin CO stripes), form (pale stripes), and motion (thick CO stripes) (DeYoe and Van Essen, 1985; Livingstone and Hubel, 1988). As with CO blobs in V1, however, we know that CO stripes in V2 cannot exist solely for the purpose of segregating color-selective cells in all primates, because owl monkeys have a similar organization of $\mathrm{CO}$ stripes in $\mathrm{V} 2$ and lack color vision (Tootell et al., 1985; our study). More recent electrophysiological and optical imaging results from the macaque monkey confirm that there is a tendency for color-selective cells to be found in thin stripes and a tendency for orientation-selective cells to be found in thick stripes (Ts'o et al., 1990; Roe and Ts'o, 1995; Roe, 2004). However, data from both the squirrel monkey and the macaque have shown that the organization of orientation selectivity and other properties is not homogenously distributed within stripes, and that the degree of selectivity and the mapping of orientation preference does not obey stripe boundaries (Roe and Ts'o, 1995; Roe and Ts'o, 1997; Ts'o et al., 2001; Roe, 2004). These data indicate that there are probably more than three functional compartments in V2 of these species, with each CO domain potentially divided into two or more compartments. Our data from the current study provide strong quantitative support for a similar type of organization in the owl monkey, revealing this type of functional organization of $\mathrm{V} 2$ to be a robust feature in simian primates. Nevertheless, the functional organization in prosimians appears to be different, because no evidence for $\mathrm{CO}$ stripes or areas of high and low orientation selectivity in V2 of the bush baby have been demonstrated (Bosking et al., 1996).

In the owl monkey, we found that at least four functional compartments exist in V2, two containing orientation-selective cells and correlating with the thick CO bands and one class of pale bands and the other two containing cells with low orientation selectivity and correlating with the CO thin bands and the second 
class of pale bands. Although the current data suggest four functional domains, it is possible that additional subdivisions of V2 would be revealed if we probed with other stimulus features such as direction of motion or disparity. In addition, strong anatomical evidence exists in cebus monkeys for four V2 compartments (Nascimento-Silva et al., 2003). Nascimento-Silva et al. (2003) identified two cell types in $\mathrm{CO}$ pale bands, those projecting to visual area PO and those projecting to V4; no cells were found that projected to both areas, suggesting two types of CO pale bands exist. Similarly, they found that area MT received exclusive input from $\mathrm{CO}$ thick bands, whereas $\mathrm{V} 4$ received input from $\mathrm{CO}$ thin bands. Thus, as in the present study, their anatomical results support the existence of at least four V2 compartments.
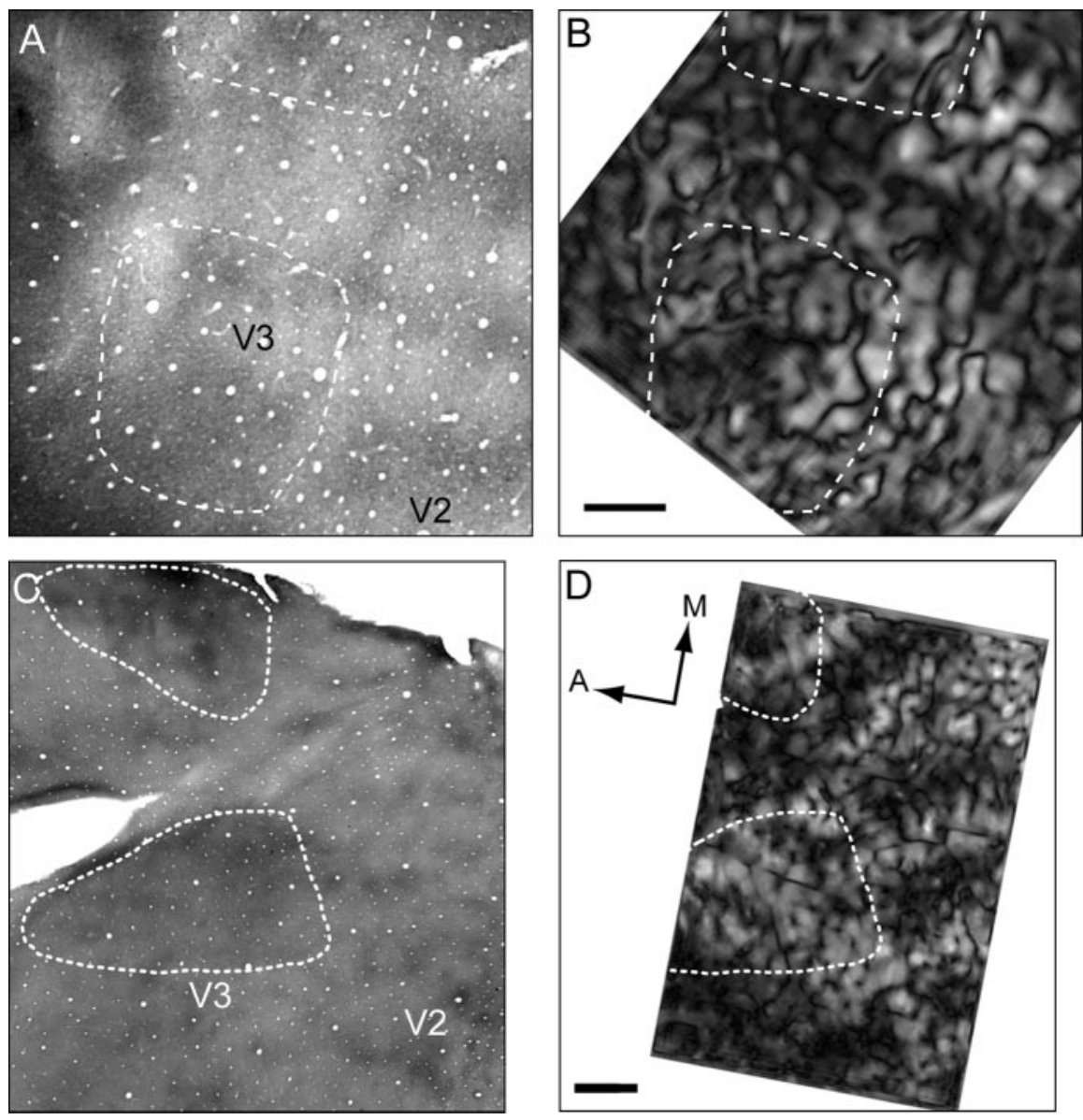

Figure 7. Comparisons between $C 0$ modules and orientation preference domains in V3. This figure presents two cases: $A$ and $B$ (case 1), $C$ and $D$ (case 2). C and $D$ are from the same case shown in Figures 1 and 2. The white dashed lines in $A$ and $C$ encircled the V3 $\mathrm{C} 0$ dense zones and were drawn based on $\mathrm{CO}$ threshold contours; these lines were transferred to the V3 orientation magnitude map in $B$ and $D$, respectively. See Results for details. Scale bars, $1 \mathrm{~mm}$.
Within the orientation-selective regions of V2, we found that the size of orientation domains was larger than that of $\mathrm{V} 1$, and that the pinwheel density was correspondingly lower. A similar increase in domain size has been observed in the squirrel monkey, bush baby, and macaque monkey (Ts'o et al., 1990; Malach et al., 1994; Bosking et al., 1996). In the owl monkey, this trend is continued in V3, which has larger domains than both V1 and V2. It is not clear why this trend toward larger functional domain sizes exists, but it may be correlated with changes in the organization of intrinsic connections within each area (Amir et al., 1993).

Finally, because optical imaging is simply based on differences in reflectance averaged over small regions of cortex, we cannot rule out alternative interpretations for areas that show low orientation selectivity in V2 and V3. The following are two other interpretations: (1) the individual cells may be well tuned to specific orientations but not spatially organized into columns that respond to similar orientations, as has been demonstrated in V1 and V2 of mice and squirrels; (2) the cells in these regions may not respond well to the grating stimuli used in our experiments. The comparable response ranges of intrinsic signal strength in regions of low and high orientation selectivity in V2 make the second possibility less likely. It is important to note, however, that whether the underlying difference in these regions is low selectivity, lack of columnar organization, or low levels of response to the grating stimuli, each of these alternatives would constitute a real difference from the other regions of V2 tested with the same stimuli.

\section{The functional organization of V3}

Although no previous optical imaging studies had examined the functional organization of $\mathrm{V} 3$, the existence of dark and pale CO domains suggested that different processing compartments might also exist in this visual area. Here, we provide evidence for two functional compartments in owl monkey V3: large zones that stain darkly for $\mathrm{CO}$ and have high orientation selectivity, separated by narrow zones that show light staining for $\mathrm{CO}$ and low orientation selectivity. Zones of high orientation selectivity in V3 had a similar geomet-

Table 2. Relationship between $\mathrm{V} 1$ size and the number of $\mathrm{C} 0$ blobs and pinwheels

\begin{tabular}{lllccr}
\hline Species & V1 size $\left(\mathrm{mm}^{2}\right)$ & CO blob density $\left(\right.$ per $\left.\mathrm{mm}^{2}\right)$ & Total CO blobs in V1 & Pinwheel density (per $\mathrm{mm}^{2}$ ) & Total pinwheels in V1 \\
\hline Bush baby (Ottolemur garnetti) & 139 & 2.8 & 382 & 6.4 & 890 \\
Owl monkey (Aotus trivirgatus) & 287 & 3.6 & 1030 & 7.4 & 2124 \\
Squirrel monkey (Saimiri sciureus) & 637 & 4.5 & 2867 & 11.0 & 7007 \\
Macaque monkey (Macaca nemestrina, & & & 5584 & 8.0 & 10152 \\
$\quad$ Macaca fasciculari, Macaca mulatta) & 1269 & 4.4 & $\mathrm{NA}$ & 9.4 & 564 \\
Tree shrew (Tupaia belangeri) & 60 & NA & 570 & 3.0 & 1140 \\
Cat (Felis domesticus) & 380 & 1.5 &
\end{tabular}

Numbers shown are means from several studies. Estimates of V1 size and CO blob density for all primates are from Condo and Casagrande (1990) (see Fig. 11 for list of references). V1 size for tree shrews is from Lyon et al. (1998); V1 size for cat is from Tusa et al. (1978). CO blob density for macaque monkey is from Murphy et al. (1998) and Livingstone and Hubel (1984); CO blob density for cat is from Boyd and Matsubara (1996). Pinwheel densities are averages calculated from the following sources: bush baby, Bosking et al. (1996); owl monkey, our study; squirrel monkey, Blasdel and Cambell (2001); macaque monkey, Obermayer and Blasdel (1997); tree shrew, Bosking et al. (1997); cat, Muller et al. (2000). There is a strong linear correlation between the total CO blobs and the total pinwheels (total pinwheels $=1.85 \times$ total C 0 blobs $+399 ; r^{2}=0.96$ ). 
ric organization to those in V2 with both pinwheels and linear zones but with larger domain sizes. Electrophysiological recordings from $\mathrm{V} 3$ in macaque monkeys have found both nonoriented cells and orientation-sensitive cells (Baizer, 1982; Adams and Zeki, 2001). Also, a 2-deoxyglucose study of orientation selectivity in macaque monkeys suggested that regions of high and low orientation selectivity may exist in V3 (Vanduffel et al., 2002). Combined with the current results, these studies suggest that the compartmental organization of V3 that we see in owl monkeys could generalize across simian primates.

\section{References}

Adams DL, Zeki S (2001) Functional organization of macaque V3 for stereoscopic depth. J Neurophysiol 86:2195-2203.

Allman J, Zucker S (1990) Cytochrome oxidase and functional coding in primate striate cortex: a hypothesis. Cold Spring Harb Symp Quant Biol 55:979-982.

Amir Y, Harel M, Malach R (1993) Cortical hierarchy reflected in the organization of intrinsic connections in macaque monkey visual cortex. J Comp Neurol 334:19-46.

Baizer JS (1982) Receptive field properties of V3 neurons in monkey. Invest Ophthalmol Visual Sci 23:87-95.

Bartfeld E, Grinvald A (1992) Relationships between orientation-preference pinwheels, cytochrome oxidase blobs, and ocular-dominance columns in primate striate cortex. Proc Natl Acad Sci USA 89:11905-11909.

Blasdel GG (1992a) Differential imaging of ocular dominance and orientation selectivity in monkey striate cortex. J Neurosci 12:3115-3138.

Blasdel GG (1992b) Orientation selectivity, preference, and continuity in monkey striate cortex. J Neurosci 12:3139-3161.

Blasdel G, Campbell D (2001) Functional retinotopy of monkey visual cortex. J Neurosci 21:8286-8301.

Blasdel GG, Salama G (1986) Voltage-sensitive dyes reveal a modular organization in monkey striate cortex. Nature 321:579-585.

Bosking LE, White LE, Casagrande VA, Fitzpatrick D (1996) Functional organization of areas V1 and V2 in the prosimian galago revealed by optical imaging. Soc Neurosci Abstr 22:1610.

Bosking WH, Zhang Y, Schofield B, Fitzpatrick D (1997) Orientation selectivity and the arrangement of horizontal connections in tree shrew striate cortex. J Neurosci 17:2112-2127.

Bosking WH, Kretz R, Pucak ML, Fitzpatrick D (2000) Functional specificity of callosal connections in tree shrew striate cortex. J Neurosci 20:2346-2359.

Boyd JD, Casagrande VA (1999) Relationships between cytochrome oxidase (CO) blobs in primate $\mathrm{V} 1$ and the distribution of neurons projecting to the middle temporal area (MT). J Comp Neurol 409:573-591.

Boyd JD, Matsubara JA (1996) Laminar and columnar patterns of geniculocortical projections in the cat: relationship to cytochrome oxidase. J Comp Neurol 365:659-682.

Casagrande VA, Kaas JH (1994) The afferent, intrinsic, and efferent connections of primary visual cortex in primates. In: Cerebral cortex, Vol 10 (Peters A, Rockland KS, eds), pp 201-259. New York: Plenum.

Chapman B, Stryker MP, Bonhoeffer T (1996) Development of orientation preference maps in ferret primary visual cortex. J Neurosci 16:6443-6453.

Condo GJ, Casagrande VA (1990) Organization of cytochrome oxidase staining in the visual cortex of nocturnal primates (Galago crassicaudatus and Galago senegalensis): I. Adult patterns. J Comp Neurol 293:632-645.

DeBruyn EJ, Casagrande VA, Beck PD, Bonds AB (1993) Visual resolution and sensitivity of single cells in the primary visual cortex (V1) of a nocturnal primate (bush baby): correlations with cortical layers and cytochrome oxidase patterns. J Neurophysiol 69:3-18.

DeYoe EA, Van Essen DC (1985) Segregation of efferent connections and receptive field properties in visual area V2 of the macaque. Nature 317:58-61.

Edwards DP, Purpura KP, Kaplan E (1995) Contrast sensitivity and spatial frequency response of primate cortical neurons in and around the cytochrome oxidase blobs. Vis Res 35:1501-1523.

Felleman DJ, Van Essen DC (1987) Receptive field properties of neurons in area V3 of macaque monkey extrastriate cortex. J Neurophysiol 57:889-920.

Galuske RA, Schmidt KE, Goebel R, Lomber SG, Payne BR (2002) The role of feedback in shaping neural representations in cat visual cortex. Proc Natl Acad Sci USA 99:17083-17088.
Gegenfurtner KR, Kiper DC, Levitt JB (1997) Functional properties of neurons in macaque area V3. J Neurophysiol 77:1906-1923.

Granquist-Fraser D, Polimeni J, Schwartz EL (2003) Physical limits to spatial resolution of optical recording: photon scatter and optical defocus. Soc Neurosci Abstr 29:125.3.

Horton JC, Hubel DH (1981) Regular patchy distribution of cytochrome oxidase staining in primary visual cortex of macaque monkey. Nature 292:762-764.

Hubel DH, Wiesel TN (1977) Ferrier lecture. Functional architecture of macaque monkey visual cortex. Proc R Soc Lond B Biol Sci 198:1-59.

Issa NP, Trepel C, Stryker MP (2000) Spatial frequency maps in cat visual cortex. J Neurosci 20:8504-8514.

Jacobs GH, Neitz M, Neitz J (1996) Mutations in S-cone pigment genes and the absence of colour vision in two species of nocturnal primate. Proc Natl Acad Sci USA 263:705-710.

Landisman CE, Ts'o DY (2002a) Color processing in macaque striate cortex: relationships to ocular dominance, cytochrome oxidase, and orientation. J Neurophysiol 87:3126-3137.

Landisman CE, Ts'o DY (2002b) Color processing in macaque striate cortex: electrophysiological properties. J Neurophysiol 87:3138-3151.

Lennie P, Krauskopf J, Sclar G (1990) Chromatic mechanisms in striate cortex of macaque. J Neurosci 10:649-669.

Leventhal AG, Thompson KG, Liu D, Zhou Y, Ault SJ (1995) Concomitant sensitivity to orientation, direction, and color of cells in layers 2, 3, and 4 of monkey striate cortex. J Neurosci 15:1808-1818.

Livingstone MS, Hubel DH (1984) Anatomy and physiology of a color system in the primate visual cortex. J Neurosci 4:309-356.

Livingstone MS, Hubel DH (1988) Segregation of form, color, movement and depth: anatomy, physiology, and perception. Science 240:740-749.

Lyon DC, Kaas JH (2001) Connectional and architectonic evidence for dorsal and ventral V3, and dorsomedial area in marmoset monkeys. J Neurosci 21:249-261.

Lyon DC, Kaas JH (2002) Evidence from V1 connections for both dorsal and ventral subdivisions of $\mathrm{V} 3$ in three species of New World monkeys. J Comp Neurol 449:281-297.

Lyon DC, Jain N, Kaas JH (1998) Cortical connections of striate and extrastriate visual areas in tree shrews. J Comp Neurol 401:109-128.

Lyon D, Xu X, Casagrande VA, Stefansic J, Shima D, Kaas JD (2002) Optical imaging reveals retinotopic organization of dorsal V3 in New World owl monkeys. Proc Natl Acad Sci USA 99:15735-15742.

Malach R, Tootell RB, Malonek D (1994) Relationship between orientation domains, cytochrome oxidase stripes, and intrinsic horizontal connections in squirrel monkey area V2. Cereb Cortex 4:151-165.

Muller T, Stetter M, Hubener M, Sengpiel F, Bonhoeffer T, Godecke I, Chapman B, Lowel S, Obermayer K (2000) An analysis of orientation and ocular dominance patterns in the visual cortex of cats and ferrets. Neural Comput 12:2573-2595.

Murphy KM, Jones DG, Fenstemaker SB, Pegado VD, Kiorpes L, Movshon JA (1998) Spacing of cytochrome oxidase blobs in visual cortex of normal and strabismic monkeys. Cereb Cortex 8:237-244.

Nascimento-Silva S, Gattass R, Fiorani Jr M, Sousa AP (2003) Three streams of visual information processing in V2 of Cebus monkey. J Comp Neurol 466:104-118.

Obermayer K, Blasdel GG (1997) Singularities in primate orientation maps. Neural Comput 9:555-575.

O’Keefe LP, Levitt JB, Kipper DC, Shapley RM, Movshon JA (1998) Functional organization of owl monkey lateral geniculate nucleus and visual cortex. J Neurophysiol 80:594-609.

Roe AW (2004) Modular complexity of area V2 in the macaque monkey. In: The primate visual system (Kaas JH, Collins CE, eds), pp 109-139. Boca Raton, FL: CRC.

Roe AW, Ts'o DY (1995) Visual topography in primate V2: multiple representation across functional stripes. J Neurosci 15:3689-3715.

Roe AW, Ts'o DY (1997) The functional architecture of area V2 in the macaque monkey. In: Extrastriate cortex in primates, Vol 12 (Rockland KS, Kaas JH, Peters A, eds), pp 295-333. New York: Plenum.

Roe AW, Ts'o DY (1999) Specificity of color connectivity between primate V1 and V2. J Neurophysiol 82:2719-2730.

Schwartz EL, Polimeni J, Granquist-Fraser D, Wood RJ (2003) The structure of singular regions in cortical orientation maps: a function of spatial blur. Soc Neurosci Abstr 29:125.4. 
Shmuel A, Grinvald A (2000) Coexistence of linear zones and pinwheels within orientation maps in cat visual cortex. Proc Natl Acad Sci USA 97:5568-5573.

Shoham D, Hubener M, Schulze S, Grinvald A, Bonhoeffer T (1997) Spatiotemporal frequency domains and their relation to cytochrome oxidase staining in cat visual cortex. Nature 385:529-533.

Sincich LC, Horton JC (2002) Divided by cytochrome oxidase: a map of the projections from V1 to V2 in macaques. Science 295:1734-1737.

Swindale NV, Shoham D, Grinvald A, Bonhoeffer T, Hubener M (2000) Visual cortex maps are optimized for uniform coverage. Nat Neurosci 3:822-826.

Tootell RB, Hamilton SL, Silverman MS (1985) Topography of cytochrome oxidase activity in owl monkey cortex. J Neurosci 5:2786-2800.

Ts'o DY, Gilbert CD (1988) The organization of chromatic and spatial interactions in the primate striate cortex. J Neurosci 8:1712-1727.

Ts'o DY, Frostig RD, Lieke EE, Grinvald A (1990) Functional organization of primate visual cortex revealed by high resolution optical imaging. Science 249:417-420.

Ts'o DY, Roe AW, Gilbert CD (2001) A hierarchy of the functional organization for color, form and disparity in primate visual area V2. Vision Res 41:1333-1349.

Tusa RJ, Palmer LA, Rosenquist AC (1978) The retinotopic organization of area 17 (striate cortex) in the cat. J Comp Neurol 177:213-235.

Vanduffel W, Tootell RBH, Schoups AA, Orban GA (2002) The organiza- tion of orientation selectivity throughout macaque visual cortex. Cereb Cortex 12:647-662.

Wikler KC, Rakic P (1990) Distribution of photoreceptor subtypes in the retina of diurnal and nocturnal primates. J Neurosci 10:3390-3401.

Xiao Y, Wang Y, Felleman DJ (2003) A spatially organized representation of colour in macaque cortical area V2. Nature 421:535-539.

Xu X (2004) Functional organization of visual cortex in bush babies and owl monkeys revealed by optical imaging of intrinsic signals. $\mathrm{PhD}$ thesis, Vanderbilt University.

Xu X, Ichida JM, Allison JD, Boyd JD, Bonds AB, Casagrande VA (2001) A comparison of Koniocellular (K), Magnocelluar (M), and Parvocellular (P) receptive field properties in the lateral geniculate nucleus (LGN) of the owl monkey (Aotus trivirgatus). J Physiol (Lond) 531:203-218.

Xu X, Boyd J, Gallucci M, Thomas A, Emeric E, Barahimi B, Stefansic J, Shima D, Melzer P, Allison J, Bonds AB, Casagrande V (2003a) Spatial frequency preference maps of primate visual cortex revealed by optical imaging of intrinsic signals. J Vision 3:107a.

Xu X, Gallucci MR, Shima DW, Stefansic JD, Thomas AM, Yokoo T, Sornborger A, Bonds AB, Casagrande $\mathrm{AB}$ (2003b) Is there any relationship between cytochrome oxidase (Co) blobs and spatial frequency preference domains in primate visual cortex? Soc Neurosci Abstr 29:701.18.

Xu X, Collins CE, Kaskan PM, Khaytin I, Kaas JH, Casagrande VA (2004) Optical imaging of visually evoked responses in prosimian primates reveals conserved features of the middle temporal visual area. Proc Natl Acad Sci USA 101:2566-2571. 